ISSN 1112-9867

Available online at

http://www.jfas.info

\title{
MONITORING AND THERMAL ANALYSIS OF CO-COMPOSTING PROCESS IN A FOREST NURSERY MODERN (TUNISIA)
}

\author{
Y. M'Sadak*, I. Saad and D. Saidi
}

Département du Génie des Systèmes Horticoles et du Milieu Naturel, Institut Supérieur Agronomique de Chott-Mariem, Université de Sousse, Tunisie

Received: 27 December 2012 / Accepted: 31 March 2013 / Published online: 30 June 2013

\begin{abstract}
The objective of this work was to study thermogenesis during the process of Co-composting to assess the possibilities of thermal destruction of the germination capacity of weeds seeds. A regular monitoring of temperature has been achieved, using a probe thermometer, at nine locations of heap crafted. The outlines of temperature profiles allowed the identification of the four phases of the process, from a temperature monitoring, which is taken twice a day. The mid-monthly monitoring of temperature showed no significant differences for the first three dates of monitoring, whatever the depth and whatever time study. The bi-monthly monitoring showed insignificant differences for the first three dates, whatever the depth of the heap and whatever the studied time. The last three dates showed insignificant differences for the time of recording, whereas for the depth, the results obtained were significant.
\end{abstract}

Keywords: Forest nursery; forestry Co-composting; thermal profile; thermogenesis; monitoring spatio-temporal.

\section{INTRODUCTION}

Dans le cadre de la modernisation des pépinières forestières en Tunisie, le premier défi consistait à trouver une solution de rechange à l'utilisation du terreau forestier en vue d'améliorer les pratiques culturales et la qualité des plants produits en pépinière forestière.

Author Correspondence, e-mail: msadak.youssef@yahoo.fr

Tel.: 0021673327 546; fax: 0021673327591.

ICID: 1040082 
Pour éviter le recours aux importations des substrats (tourbe, vermiculite, perlite, ...), une attention particulière a été accordée au compostage de la biomasse forestière (écorces de pins, branches d'acacia et de maquis) en pépinière forestière [1].

Le compostage est un procédé biologique contrôlé de conversion et de valorisation des matières organiques qui procure un produit stabilisé, hygiénisé et riche en composés humiques [2;3], essentiel pour les activités agricoles (maraîchage, horticulture, grandes cultures, ...). Ce processus oxydatif, caractérisé par un dégagement de chaleur, est l'une des quelques solutions actuelles pour valoriser les résidus et déchets organiques, avec la biométhanisation [2].

Le Co-compostage ou compostage conjoint consiste à mettre dans le tas de compost des déchets de natures physique et chimique différentes pour assurer une complémentarité entre les apports en éléments minéraux. Cette technique vise à assurer l'équilibre nutritionnel aux microorganismes qui vont transformer la matière organique brute en compost de qualité [4]. Cet équilibre nutritionnel ne peut être assuré que si les quatre paramètres essentiels du processus de compostage seront présents; la porosité, la fermentation, l'agent structurant (Carbone) et l'agent fermentescible (Azote) $[2 ; 3 ; 4]$.

Le compost pur de broyat d'Acacia ou compost sylvicole est généralement préparé en ajoutant du nitrate d'ammonium $\left(\mathrm{NH}_{4} \mathrm{NO}_{3}\right)$ comme support azoté lors de la confection des andains et lors du premier retournement [1]. Parmi les supports azotés éventuels à insérer, le broyat sylvicole (riche en cellulose et en lignine) peut être associé avec le fumier ovin brut à raison d'un volume inférieur à $50 \%$ pour aboutir à la formation du Co-compost sylvicole. Si le volume de fumier incorporé dépasse $50 \%$, le produit est désigné Co-compost ovin.

Le présent travail se propose d'étudier, à titre expérimental, le processus de Co-compostage sylvicole, mis en œuvre sur la plateforme bétonnée de compostage de la pépinière forestière moderne de Chott-Mariem (Sousse- Tunisie), afin d'analyser le profil thermique, relevé à partir du suivi spatio-temporel de la température à l'intérieur du tas confectionné, à l'aide d'un thermomètre à sonde. Une telle analyse pourrait affiner davantage l'expérimentation envisagée de la destruction par le compostage de la viabilité des semences de Morelle Jaune, plante envahissante assez répandue dans certaines régions de la Tunisie Centrale (Kairouan, Sidi Bouzid, ...).

\section{MATERIEL ET METHODES}

\section{Matériel}




\section{Matériaux mis en compostage}

Le Co-compost sylvicole (CCS) produit est composé de 2/3 de broyat de branches fraîches d'Acacia cyanophylla $(\mathrm{C} / \mathrm{N}=42)$ issu d'un double broyage séparé (à couteaux, ensuite à marteaux) et de $1 / 3$ fumier ovin sans litière $(\mathrm{C} / \mathrm{N}=16)$. Il a subi quatre retournements échelonnés toutes les trois semaines. L'humidification du tas a été réalisée au départ lors de la confection du tas et lors des retournements réalisés. Le volume du tas confectionné était de 3 $\mathrm{m}^{3}$ (1,5 $\mathrm{m}$ de largeur, $2 \mathrm{~m}$ de longueur et $1 \mathrm{~m}$ de hauteur). Ce tas a été confectionné lors de la saison estivale de l'année 2007.

\section{Autre matériel}

Un thermomètre à sonde de longueur $90 \mathrm{~cm}$ a été utilisé pour mesurer périodiquement la température dans le tas de Co-compost sylvicole.

\section{METHODE}

\section{Suivi de l'évolution de la température dans le tas de Co-compost}

Le relevé de la température a constitué le meilleur moyen de suivi de la conduite de l'opération de Co-compostage. C'est lui qui détermine généralement le moment de retournement du tas. En effet, une chute de température est un indice de ralentissement des activités bactériennes dû à une insuffisance d'oxygène. Un suivi spatio-temporel régulier de la température a été accompli pour le tas de Co-compost considéré, d'une part, tous les deux jours, durant toute la période de Co-compostage à la même profondeur $(50 \mathrm{~cm})$ et à heure fixe de la journée ( 9 h du matin) au niveau de neuf endroits du tas, et d'autre part, tous les 15 jours, à trois profondeurs différentes $(10 \mathrm{~cm}, 50 \mathrm{~cm}$ et $90 \mathrm{~cm})$ et à trois horaires de la journée $(9 \mathrm{~h}, 11 \mathrm{~h}$ et $15 \mathrm{~h})$ au niveau des mêmes points de relevé (Figure 1). Le thermomètre à sonde est introduit dans les neufs endroits considérés du tas selon la profondeur désirée. 


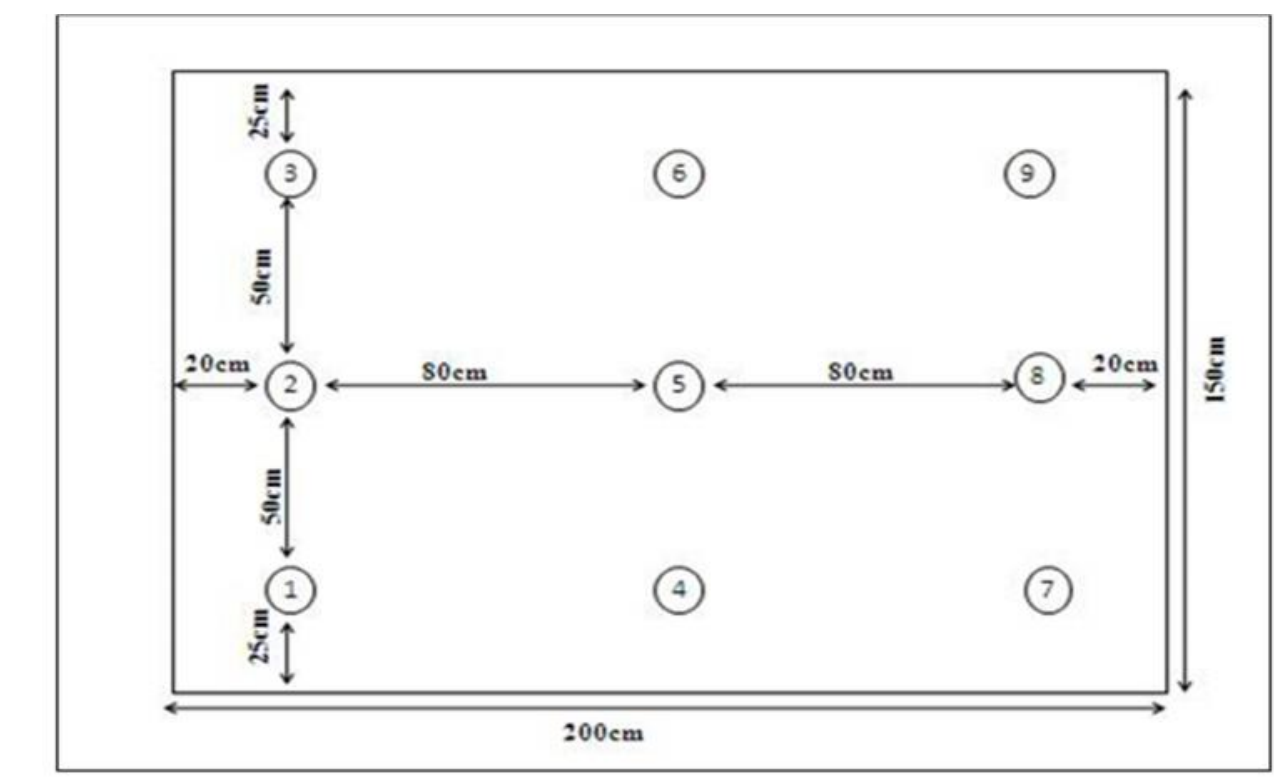

Point de relevé de température

Fig.1. Disposition des points de relevés de températures dans le tas de Co-compost

\section{Traçage des courbes d'égale température}

Le logiciel utilisé pour le traçage des courbes d'égale température ou isothermes est le SURFER (version 7).

Quatre dates, relatives aux phases du processus de compostage, ont été choisies pour le traçage des isothermes afin d'étudier l'évolution de la température à la fois spatiale et temporelle à profondeur fixe du tas $(50 \mathrm{~cm})$.

Ces dates sont relatées ci-après : 22/08/07 (phase 1), 24/08/07 (phase 2), 23/10/07 (phase 3) et 20/11/07 (phase 4).

\section{Analyse statistique}

L'analyse de la variance (ANOVA) a été réalisée par le logiciel «SPSS for Windows version 11.0 » et la comparaison des moyennes a été réalisée par les tests S.N.K. au seuil $5 \%$. L'analyse a concerné l'effet de la profondeur du tas, l'horaire de la journée de relevé de température et la position du point de relevé par rapport au centre du tas. La position gauche (g) est relative aux points relevés de température 1, 2 et 3, la position centre (c) concerne les points 4, 5 et 6 et la position droite (d) est relative aux points 7, 8 et 9 (Figure 1). 
L'analyse statistique a été réalisée séparément pour six autres dates relatées ci-après: 28/08/07 (date 1), 14/09/07 (date 2), 12/10/07 (date 3), 26/10/07 (date 4), 09/11/07 (date 5) et 23/11/07 (date 6).

Les résultats ont été présentés sous forme d'histogrammes tout en illustrant la moyenne et l'erreur standard à la moyenne (ESM).

\section{RESULTATS ET DISCUSSION}

Tracé et interprétation des courbes d'égale température relevée à la même profondeur Phase mésophile

La figure 2 illustre l'évolution de la température dans le compost après 3 jours de mise en tas (phase 1) à la même profondeur de $50 \mathrm{~cm}$. Dès le troisième jour de compostage, la température s'étale entre $21^{\circ} \mathrm{C}$ et $31^{\circ} \mathrm{C}$. Cette montée de température rapide est due en fait à la présence de la matière verte $[1 ; 2 ; 5]$.

L'écart de température est remarquable depuis les extrémités gauche et droite du tas, on note un décalage de $10^{\circ} \mathrm{C}$. Cette variabilité de température mesurée à la même profondeur du tas peut être expliquée par l'installation d'une première catégorie de microorganismes responsables de la dégradation de la matière organique, les bactéries et champignons mésophiles [4].

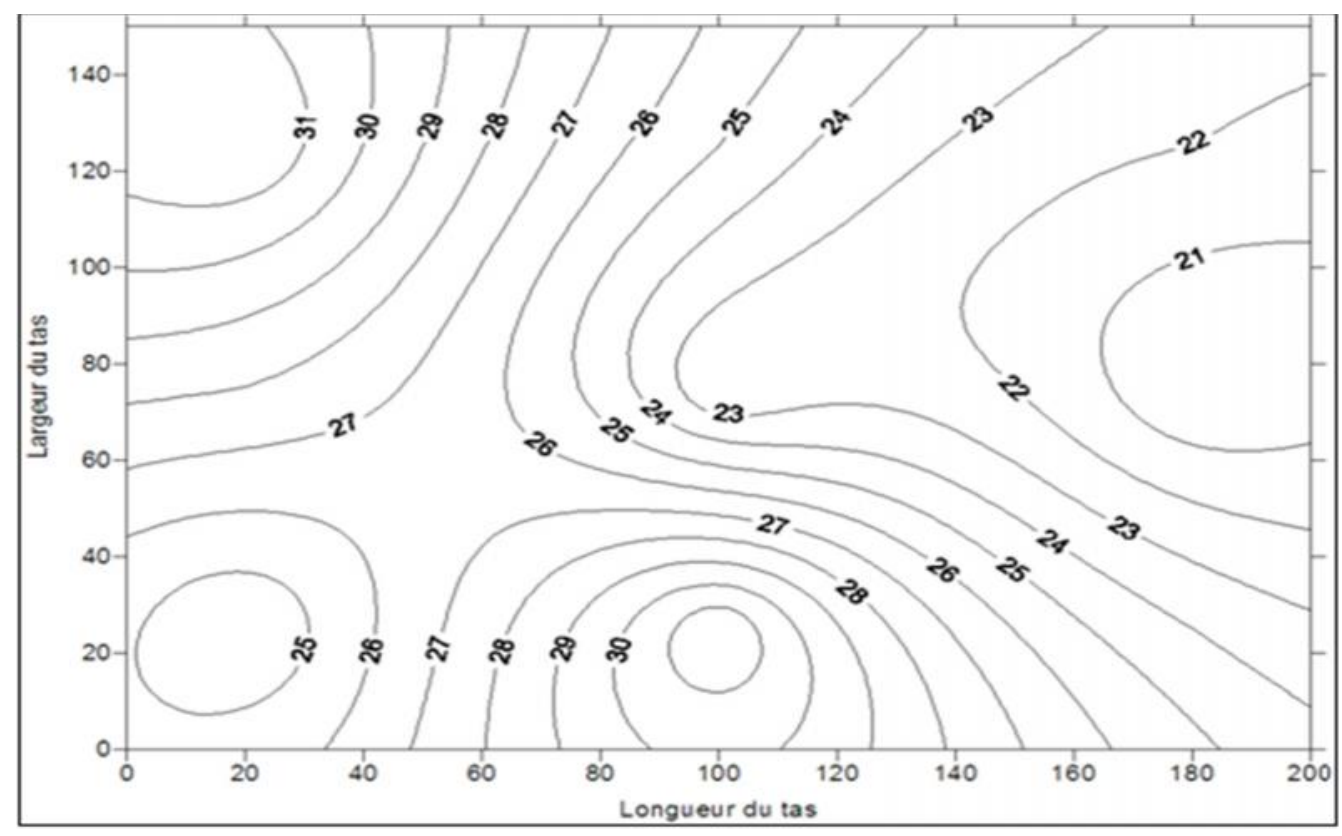

Fig.2. Courbes d'égale température de la phase mésophile du processus de compostage

\section{Phase thermophile}


La phase de dégradation est constituée de deux étapes; une mésophile atteinte entre une température de 20 à $35^{\circ} \mathrm{C}$ et une thermophile atteinte entre 35 et $70{ }^{\circ} \mathrm{C}[2 ; 5]$.

Dès le cinquième jour, la phase thermophile est déclenchée (figure 3). Cette phase est la plus longue du processus de compostage, elle a duré environ deux mois (maturité préliminaire atteinte après trois mois), toutefois, au cours de cette phase, les trois retournements réalisés tous les 21 jours ont contribué au maintien de ces conditions thermiques prolongées.

Durant cette période correspondant à la phase 2, on a noté un maximum de température atteignant $64{ }^{\circ} \mathrm{C}$. Cette température maximale a été enregistrée au centre du tas de compost où l'on observe une concentration des courbes d'égale température (figure 3). Par contre, la température est plus faible aux extrémités (courbes d'égale température dispersées). En effet, les microorganismes thermophiles responsables de la dégradation de la matière organique sont généralement plus abondants au centre qu'aux extrémités du tas.

Pour accélérer le processus de décomposition de la matière organique, il suffit de maintenir la température du tas dans l'intervalle correspondant à ces deux phases (mésophile et thermophile) pendant lesquelles la dégradation est dominante [2].

Notons enfin que la température maximale du compost est intimement liée à la matière à composter, au ratio $\mathrm{C} / \mathrm{N}$ et à l'humidité du compost $[2 ; 4 ; 5]$.

Une telle affirmation montre bien l'intérêt du Co-compostage sylvicole dans l'amélioration du rapport $\mathrm{C} / \mathrm{N}$, en associant le broyat lignocellulosique, riche en carbone (Rôle structurant) avec le fumier comme support azoté (Fermentescible).

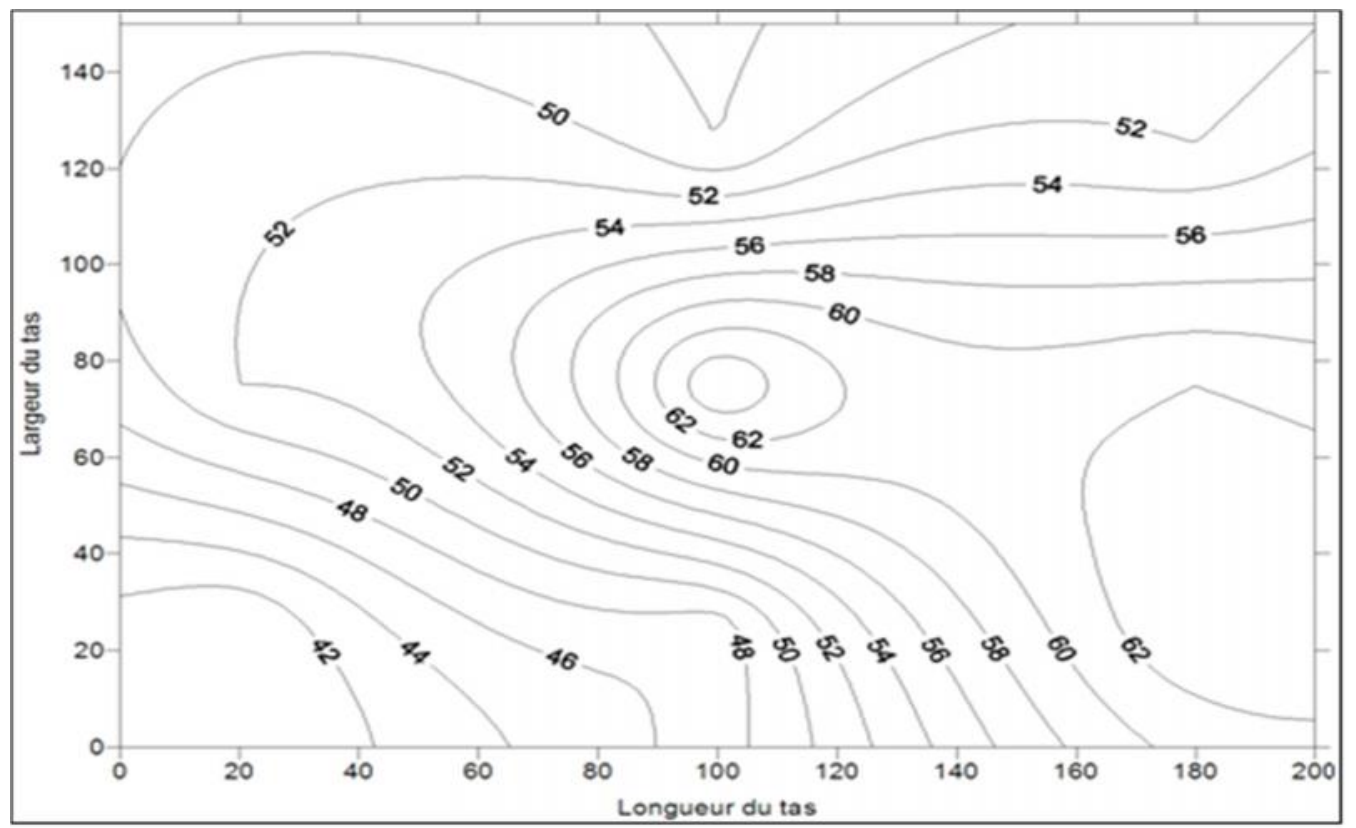

Fig.3. Courbes d'égale température de la phase thermophile du processus de compostage 


\section{Phase de refroidissement}

La figure 4 illustre l'évolution de la température dans le compost après deux mois de compostage (phase 3). Durant cette phase, on a noté une chute de température s'étalant entre $17{ }^{\circ} \mathrm{C}$ et $24{ }^{\circ} \mathrm{C}$. Il y a eu colonisation du milieu de nouveau par des microorganismes mésophiles [6]. Cette phase est en fait intermédiaire entre la phase thermophile et celle de maturation, elle prend fin avec le retour vers la température ambiante $[7 ; 8]$.

Un quatrième retournement a été effectué durant cette phase pour s'assurer de la stabilité de la température du compost, en effet, la température ne monte plus, mais au contraire, elle continue à diminuer compte tenu du changement des conditions climatiques (Passage de la saison estivale à la saison automnale).

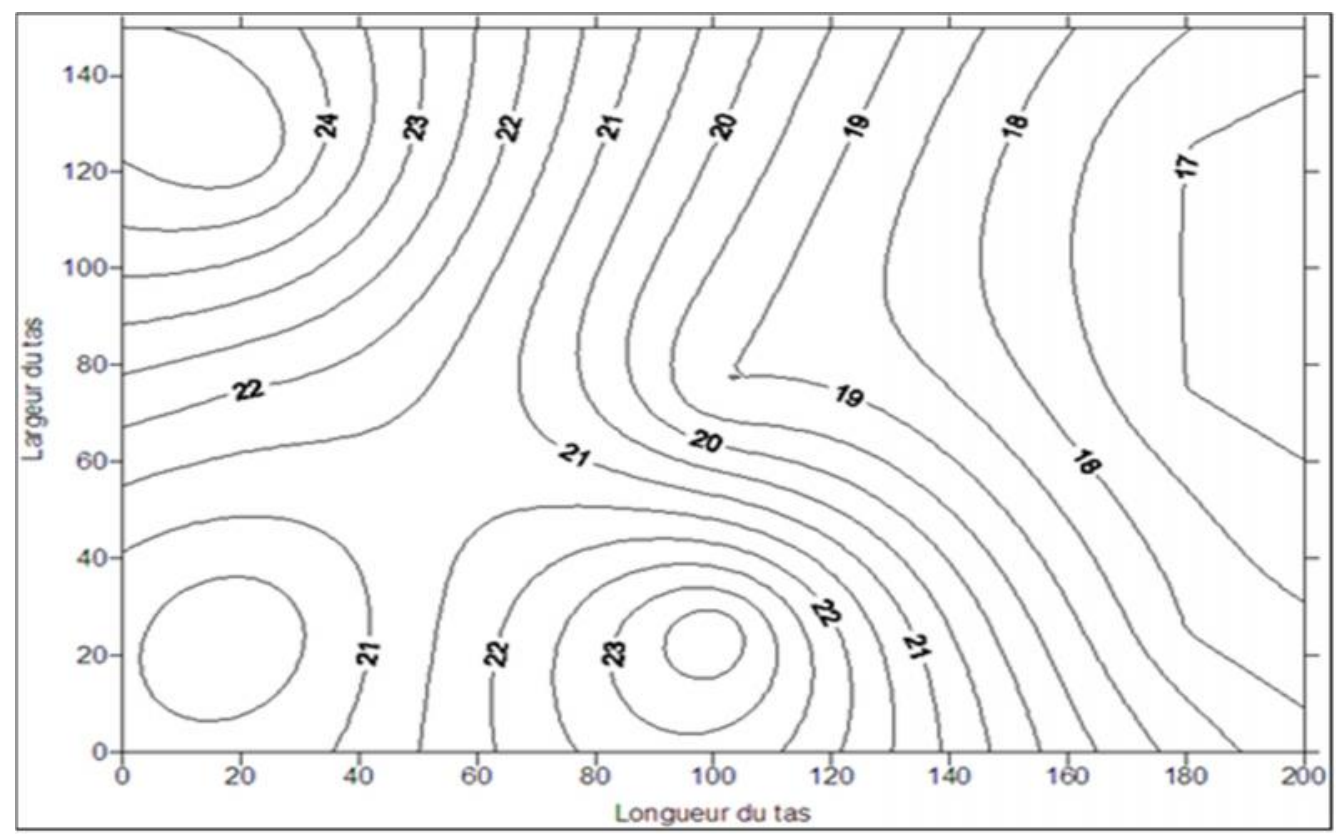

Fig.4. Courbes d'égale température de la phase de refroidissement du processus de compostage

\section{Phase de maturation}

Après la réalisation du dernier retournement, la température diminue et se stabilise à l'intervalle de $12{ }^{\circ} \mathrm{C}$ à $14{ }^{\circ} \mathrm{C}$ (figure 5). Cette date correspondant à la phase 4 présente peu d'activité microbiologique, elle est plutôt adaptée à la colonisation par les macroorganismes, essentiellement les lombrics $[7 ; 8]$. Les courbes d'égale température ont une allure similaire et la température enregistrée est presque identique dans tous les endroits du tas. Le Cocompost ainsi produit n'est pas totalement mûr, mais il est en cours de maturation. Le 
compost à maturité ne s'échauffe plus lors du retournement; il possède une température inférieure ou égale à $30{ }^{\circ} \mathrm{C}$ et n'est pas phytotoxique [9]. Le ratio $\mathrm{C} / \mathrm{N}$ et l'humidité relative du substrat initial jouent un rôle primordial dans la réussite du compostage correspondant à la dégradation aérobie des déchets organiques biodégradables. D'après [10], le compost devrait être produit par un processus qui combine au départ des matériaux végétaux et animaux, avec un ratio $\mathrm{C} / \mathrm{N}$ initial compris entre $25 / 1$ et $40 / 1$. L'excès d'humidité, s'il est supérieur à $75 \%$ inhibe le démarrage rapide du processus de compostage [11]. Le taux d'humidité (ou le pourcentage de matière sèche) a une influence majeure sur le taux de décomposition et la tendance à la stabilisation, puisque la chaleur métabolique générée pendant le processus provoque l'évaporation de l'eau. Les facteurs qui contribuent à la perte d'humidité incluent l'évaporation, le lessivage et l'aération, naturelle ou forcée [12].

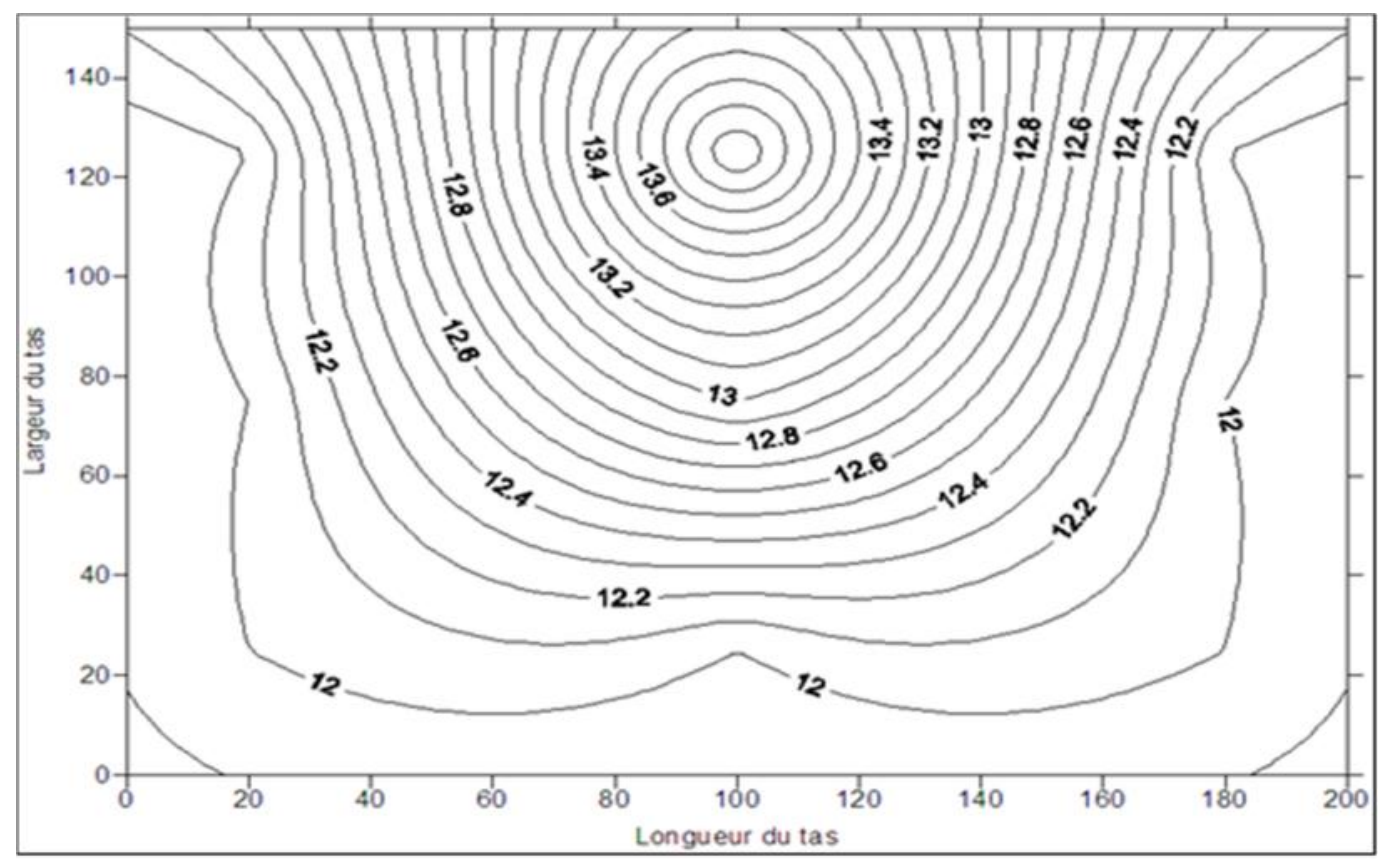

Fig.5. Courbes d'égale température de la phase de maturation du processus de compostage

\section{Analyse statistique des résultats thermiques relevés}

\section{Evolution de la température dans le tas selon différentes profondeurs}

L'analyse statistique a montré des différences significatives entre les traitements effectués $(\mathrm{P}<0,001)$ respectivement pour les dates 4, 5 et 6 (figure 6, à titre d'exemple). Par ailleurs, la comparaison des moyennes selon les tests S.N.K. a permis de dégager un nombre différent de sous-ensembles pour les traitements effectués au seuil de $5 \%$. 
Dans l'ensemble, le nombre de sous-classes obtenu lors de l'étude de l'effet de la profondeur était de deux, une classe pour $10 \mathrm{~cm}$ et une classe pour $50 \mathrm{~cm}$ et $90 \mathrm{~cm}$. En effet, plus la profondeur est importante, plus la température est élevée.

L'analyse statistique a été effectuée pour l'évolution de la température suivant la position dans le plan (Position xy) selon les différentes profondeurs (z) pour pouvoir apprécier sa répartition dans le tas suivant toutes les directions ( $\mathrm{x}, \mathrm{y}$ et $\mathrm{z}$ ). Le résultat était significatif seulement pour la date 4 (figure 6).

En général, les valeurs maximales de température ont été relevées à mi- profondeur $(50 \mathrm{~cm})$ et au centre du tas, lieu de multiplication la plus importante de microorganismes thermophiles et mésophiles. Ces augmentations de température sont le résultat de la dégradation de la matière organique due à l'activité des microorganismes $[2 ; 5]$.

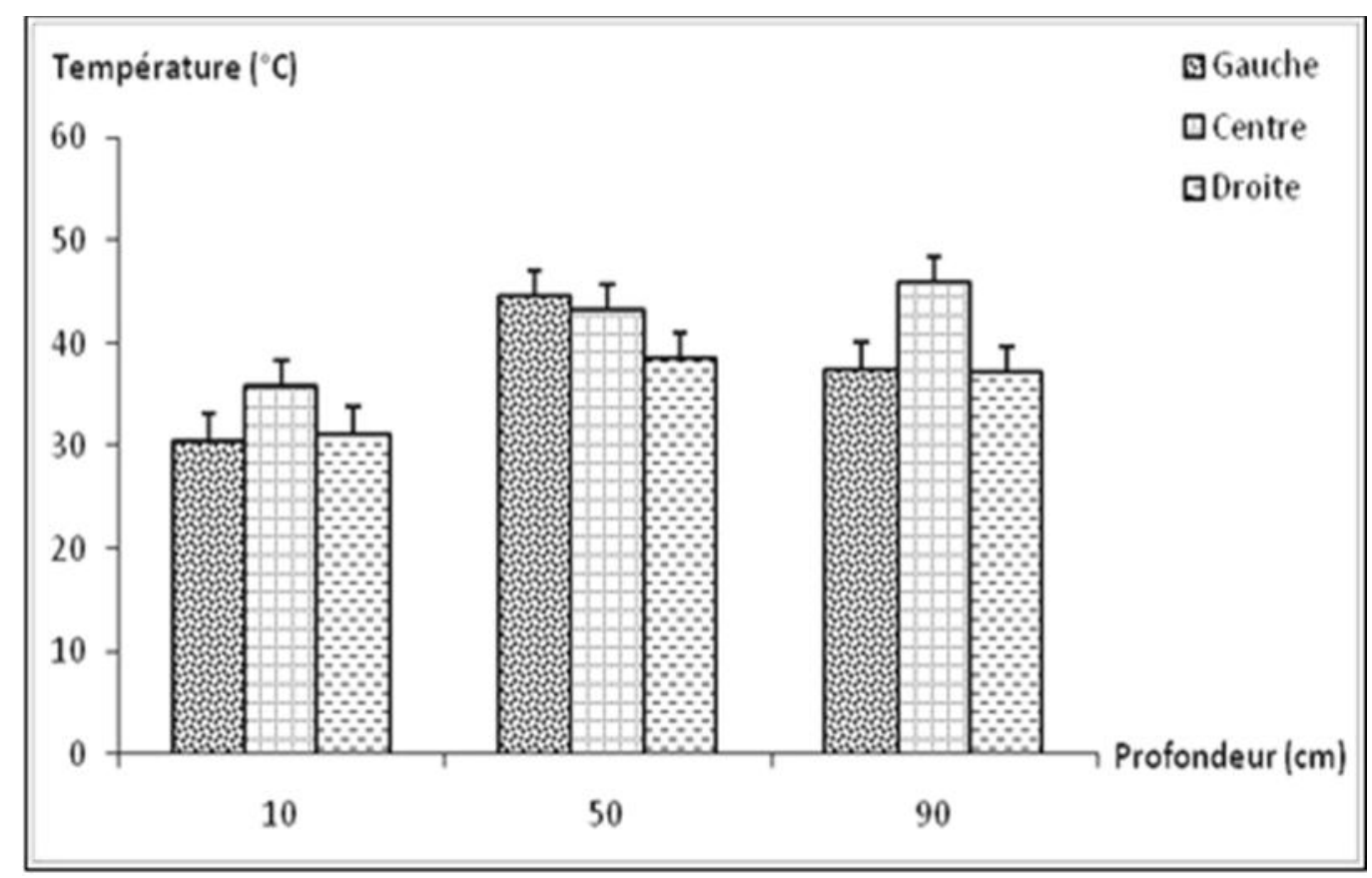

Fig.6. Variation de la température moyenne en fonction de la profondeur du tas (Date 4)

\section{Evolution de la température dans le tas suivant différents horaires de la journée}

L'analyse statistique a montré des différences non significatives entre les traitements effectués $(\mathrm{P}<0,001)$ à la profondeur de $50 \mathrm{~cm}$ (Tableau 1).

Tableau 1. Récapitulatif des effets non significatifs des traitements statistiques réalisés

Date 1 Date 2 Date 3 Date 4 Date 5 Date 6

Traitement 1 Profondeur * * * *




\begin{tabular}{|c|c|c|c|c|c|c|c|}
\hline & Position xy & $*$ & $*$ & $*$ & & $*$ & $*$ \\
\hline \multirow{2}{*}{ Traitement 2} & Heure & $*$ & $*$ & $*$ & $*$ & $*$ & $*$ \\
\hline & Position xy & $*$ & $*$ & $*$ & & & $*$ \\
\hline
\end{tabular}

$* \overline{\text { Effet non significatif }}$

La répartition de la température est homogène à mi- profondeur du tas à n'importe quelle heure étudiée de la journée (figure 7, à titre d'exemple). Un tel résultat pourrait être expliqué surtout par les variations de température ambiante relativement faibles entre $9 \mathrm{~h}$ et $15 \mathrm{~h}$ de la journée durant la majorité de la période du suivi caractérisée par une forte chaleur.

Toutefois, les différences étaient significatives pour certaines dates (dates 4 et 5) pour l'analyse selon la position xy. En effet, la température maximale était enregistrée pour les deux dates à mi- profondeur et au centre du tas, selon toutes les directions (x, y et z), et à n'importe quelle heure de relevé de température.

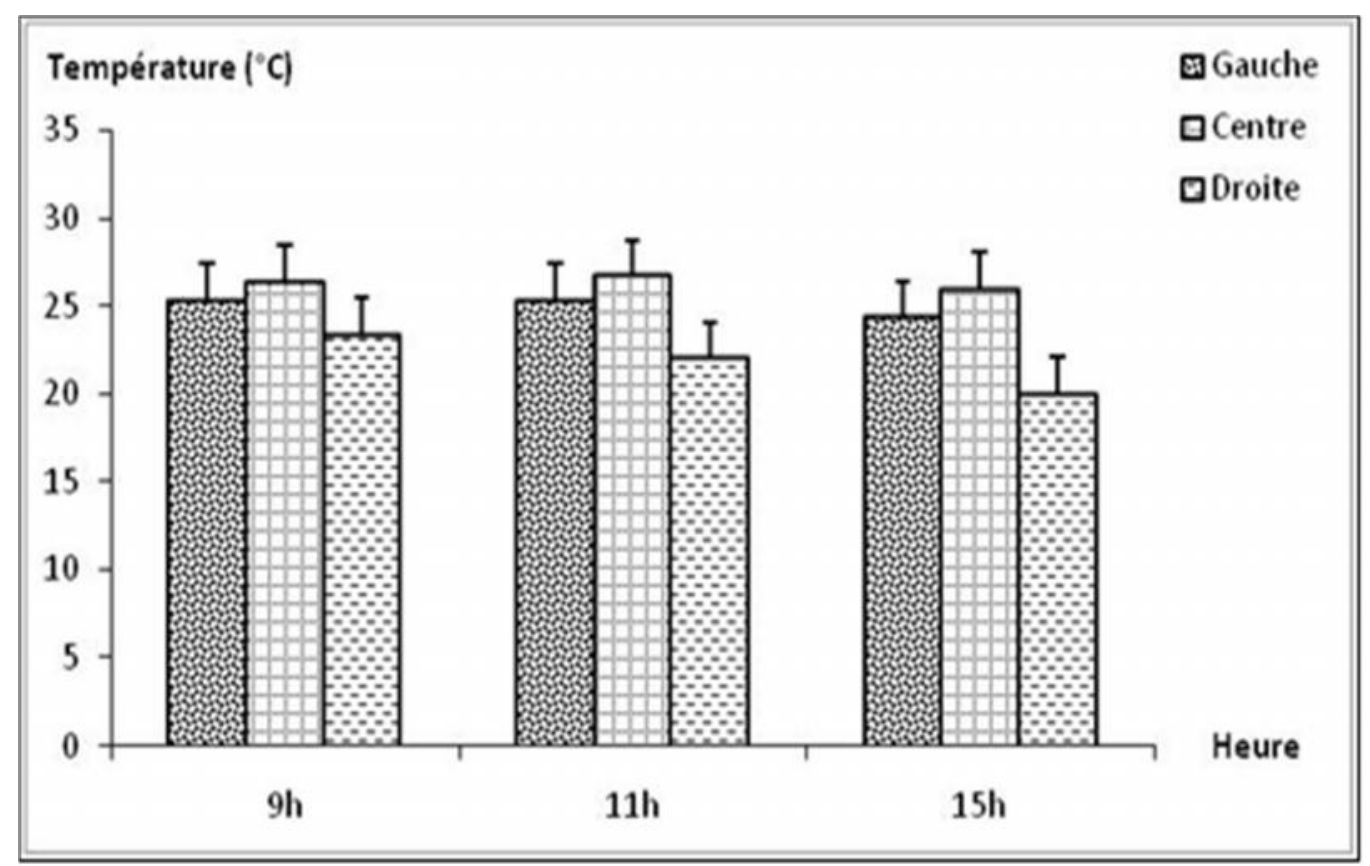

Fig.7. Variation de la température moyenne en fonction de l'heure de relevé (Date 5)

\section{Autres résultats thermiques}

L'analyse statistique de différents traitements effectués a donné des différences non significatives pour d'autres dates (tableau 1). Un tel résultat pourrait être expliqué par la coïncidence des retournements réalisés avec les dates en question et éventuellement par l'effet des conditions climatiques durant la période considérée (tas de compost non abrité). 
Pour une meilleure analyse thermique, des enregistrements de température devraient substituer les relevés périodiques. Pour cela, le système Thermo-bouton $₫$ constitue une bonne alternative pour le contrôle en temps réel et l'enregistrement en continu de la température [13].

\section{CONCLUSION}

Les courbes d'égale température tracées à partir des relevés biquotidiens de température à $50 \mathrm{~cm}$ de profondeur du tas et à $9 \mathrm{~h}$ du matin ont permis d'étudier la répartition de la température dans les différents endroits du Co-compost sylvicole. Les dates choisies pour le traçage de ces courbes ont permis d'étudier l'évolution temporelle de température dans le tas et de mettre en relief les différentes phases du processus de compostage.

L'analyse statistique des relevés mi- mensuels de température en fonction de la profondeur du tas était significative pour certaines dates seulement.

La température maximale était relevée au centre et à mi- profondeur du tas, lieu favorable pour la multiplication des microorganismes thermophiles responsables de la dégradation de la matière organique.

L'analyse statistique des relevés de température relatifs à différents horaires de la journée était non significative pour toutes les dates étudiées.

L'effet de la position du tas (gauche, centre et droite) s'est montré significatif uniquement pour la date 4 en l'étudiant selon la profondeur, alors qu'il était significatif pour cette même date et pour la date suivante en l'étudiant selon l'horaire de relevé.

Dans l'avenir, il convient d'analyser les profils thermique et/ou hygrométrique dans un tas ou dans un andain de compost en ayant recours à des relevés internes à l'aide des thermo-boutons ou des hygro-boutons. De tels systèmes de contrôle en temps réel, facilitant largement la collecte des données (stockage et traitement informatique ultérieur) tout en améliorant la précision, méritent d'être acquis pour des expérimentations futures.

\section{REMERCIEMENTS}

Ce travail n'a été commode que grâce à la participation de la Pépinière Forestière Moderne de Chott-Mariem (Sousse, Tunisie) qui a placé à notre disposition notamment une partie de sa plate-forme de compostage et tous les moyens nécessaires pour mettre en œuvre l'expérimentation (broyage de la biomasse sylvicole, suivi thermique interne du tas de compost, ...). 


\section{REFERENCES BIBIOGRAPHIQUES}

[1] Ammari Y., Lamhamedi M.S., Akrimi N. et Zine El Abidine A. Compostage de la biomasse forestière et son utilisation comme substrat de croissance pour la production de plants en pépinières forestières modernes. Revue de l'I.N.A.T., 2003, 18 (2), 99-119.

[2] Mustin M. Le compost: Gestion de la matière organique. Ed. François Dubusc, Paris, 1987, $954 \mathrm{p}$.

[3] Haug R.T. The practical handbook of compost engineering. Lewis Publishers, 1993, $717 \mathrm{p}$.

[4] Stoffella P.J. and Kahn B.A. Compost utilization in horticultural cropping Systems. Lewis Publishers, New York, USA, 2001, 413 p.

[5] Dalzell H.W., Biddlestone A.J., Gray K.R. and Thurairajan K. Soil management: compost production and use in tropical and subtropical environments. FAO Soils Bulletin 56. Rome: Food and Agriculture Organization of the United Nations, 1988, 177 p.

[6] Leclerc B. Guide des matières organiques. Deuxième édition. ITAB, France. Tome 1, 2001, 73-108.

[7] Godden B. La gestion des effluents d'élevage. Techniques et aspects du compostage dans une ferme biologique. Revue de l'Ecologie, 1995, 13, p. 37.

[8] Gobat J.M., Aragno M. et Matthey W. Le sol vivant. Bases de la pédologie. Biologie des sols. Presses Polytechniques et Universitaires Romandes, Lausanne, Suisse Collection « Gérer l'environnement », 1998, 14, 519 p.

[9] Baltazart A. Propriétés Physiques, Chimiques, Biologiques et Nutritives des Litières en Élevage de Volailles. Thèse Vétérinaire, E.N.V. Alfort, France, 2010, 184 p.

[10] Ferguson J.J., Ziegler M.R. Guidelines for purchase and application of poultry manure for organic crop production. Forida Cooperative Extension Service, Institute of Food and Agricultural Sciences, April 2004. En ligne, Gainesville (Floride, Etats-Unis d'Amérique) : University of Florida, publication HS973. [http://edis.ifas.ufl.edu].

[11] Georgakakis D., Krintas T. Optimal use of the Hosoya system in composting poultry manure. Bioresource Technology, 2000, 72, 227-233.

[12] Kelleher B.P., Leahy J.J., Henihan A.M., O'Dwyer T.F., Sutton D., Leahy M.J. Advances in poultry litter disposal technology - a review. Bioresource Technology, 2002, 83(1), 27-36. 
[13] Sandrin Gabriel-Robez E., Brugere H. Maîtrise du risque sanitaire dans une unité de compostage de sous-produits animaux - Mise en application du règlement CE 1774/2002 modifié. Proceeding Renc. Rech. Ruminants, 2007, 14, 53-56.

[www.journees3r.fr/IMG/.../2007_01_environnement_09_Sandrin.pd...].

\section{How to cite this article}

M'Sadak Y, Saad I and Saidi D. Monitoring and thermal analysis of co-composting process in a forest nursery modern (Tunisia). J Fundam Appl Sci. 2013, 5(1), 1-13. 